\title{
Use of daptomycin in the treatment of vancomycin-resistant enterococcal urinary tract infections: a short case series
}

\author{
Divya Pradeep Ramaswamy ${ }^{1,4}$, Maria Amodio-Groton² and Stephen J Scholand ${ }^{3,5^{*}}$
}

\begin{abstract}
Background: Vancomycin-resistant enterococci are a leading cause of hospital-acquired urinary tract infection and a growing concern for the clinician. The aim of this study was to evaluate the effectiveness of daptomycin in the treatment of patients with vancomycin-resistant enterococcal urinary tract infection treated in our 200-bed community-based institution.
\end{abstract}

Methods: Patients with confirmed symptomatic vancomycin-resistant enterococcal urinary tract infection identified by infectious disease consultation between January 1, 2007, and December 8, 2009, vancomycin-resistant enterococci-positive urine culture, and urinary symptoms and/or pyuria on urinalysis, and treated with daptomycin, were included in this case series. Daptomycin was generally administered at a planned dosage regimen of $\geq 5 \mathrm{mg} / \mathrm{kg}$ every 24 hours in patients with normal to moderately impaired kidney function or every 48 hours in patients with severe kidney disease. Microbiologic cure was defined as eradication of vancomycin-resistant enterococci in urine cultures taken after the completion of daptomycin treatment. Clinical cure was defined by symptom resolution, as assessed by the infectious disease clinician caring for the patient.

Results: Included in this case series are 10 patients who received daptomycin for confirmed vancomycin-resistant enterococcal urinary tract infection. Patients had a history of extensive hospital stays. Chart review revealed that all levels of kidney function (3, 2, 3, and 2 patients with kidney disease classified as normal, mild, moderate, and severe/kidney failure, respectively) were represented in the sample and that patients with $(n=5)$ or without $(n=5)$ previous urinary tract infection and with $(n=3)$ or without $(n=7)$ Foley catheters were included. Treatment with daptomycin achieved clinical cure and vancomycin-resistant enterococcal eradication in all cases in this series.

Conclusion: Treatment with daptomycin was well tolerated and effective in all patients in this series, regardless of renal function, history of urinary tract infection, or Foley catheter use. This study adds to emerging clinical evidence that daptomycin is a valuable treatment for vancomycin-resistant enterococcal urinary tract infection.

Keywords: Daptomycin, Enterococcus faecalis, Enterococcus faecium, Urinary tract infection, Vancomycin-resistant enterococci

\section{Background}

In the United States, the Gram-positive bacterium Enterococcus accounts for $12 \%$ of all cases of hospitalacquired infection and is most often implicated in urinary tract infections (UTIs) [1-3]. Recent data show that approximately $33 \%$ of all clinical enterococcal isolates in

\footnotetext{
*Correspondence: rabiesfreeworld@yahoo.com

${ }^{3}$ Division of Infectious Diseases, St. Mary's Hospital, Waterbury, Connecticut 06706, USA

${ }^{5}$ Current affiliations: Cornell Scott Hill Health Center, New Haven, Connecticut 06519, USA

Full list of author information is available at the end of the article
}

the United States are vancomycin-resistant enterococci (VRE) [2]. In North America, VRE are mainly derived from the species Enterococcus faecium (92.8\%) and Enterococcus faecalis (6.7\%) [4]. In the past decade, VRE have become increasingly involved in nosocomial infections in the United States [5,6], which has resulted in excessive morbidity, mortality, and health care costs [7]. Nearly $10 \%$ of all urinary enterococcal isolates in the United States are VRE; most of these are also E. faecium (88.4\%) [3].

Because VRE, particularly E. faecium strains, exhibit resistance to many antimicrobials traditionally used to target

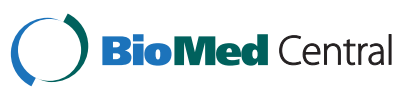


vancomycin-susceptible isolates, the treatment of patients with VRE UTIs remains a challenge for the clinician [8]. Indeed, no drugs are approved by the US Food and Drug Administration (FDA) for the treatment of patients with VRE UTIs, and reliable clinical data about the optimal management of VRE UTIs are lacking in the scientific literature [7]. Among the therapeutic options to be considered are those that have demonstrated activity against VRE in vitro, including older agents such as doxycycline, fosfomycin, and nitrofurantoin and newer agents such as daptomycin, linezolid, quinupristin-dalfopristin, and tigecycline [3,7,9].

Daptomycin is a bactericidal cyclic lipopeptide approved by the FDA for the management of complicated skin and skin structure infections caused by susceptible isolates of a variety of Gram-positive bacteria, including methicillinresistant Staphylococcus aureus, and for the management of bacteremia, including that associated with right-sided infective endocarditis, caused by methicillin-susceptible and methicillin-resistant isolates of $S$. aureus [10]. Although the approved use of daptomycin in enterococcal infections is limited to the treatment of patients with complicated skin and skin structure infections caused by vancomycin-susceptible isolates of E. faecalis [10], 100\% and $99.7 \%$ of vancomycin-resistant E. faecalis and $E$. faecium, respectively, were susceptible to daptomycin in more than 700 nonurinary VRE strains collected in the United States between 2007 and 2008 [11]. The daptomycin minimal inhibitory concentration required for $90 \%$ inhibition $\left(\mathrm{MIC}_{90}\right)$ of vancomycin-resistant $E$. faecalis was determined to be $1 \mu \mathrm{g} / \mathrm{mL}$, whereas that for vancomycin-resistant $E$. faecium was $2 \mu \mathrm{g} / \mathrm{mL}$ [11]. The use of daptomycin is a particularly promising pharmacotherapeutic approach against VRE UTIs because $50 \%$ to $70 \%$ of the dose is excreted unchanged in the urine 24 hours after intravenous administration compared with $30 \%$ to $40 \%$ for linezolid, $15 \%$ to $19 \%$ for quinupristindalfopristin, and $20 \%$ to $30 \%$ for tigecycline [7]. Moreover, another case series has described the successful use of daptomycin in patients with VRE UTIs [12].

In light of the scarcity of treatment options for VRE UTI and clinical data related to daptomycin use in this setting, the purpose of this report is to describe the clinical experience with daptomycin as part of the management of VRE UTIs in our acute care hospital. Our objective is to offer further evidence supporting daptomycin as a viable approach to managing a continuing therapeutic challenge.

\section{Methods}

This study was conducted at St. Mary's Hospital, a small community-based institution with approximately 200 beds, serving an urban population in Waterbury, Connecticut. Our catchment area included a number of nursing homes that contributed up to one-third of our inpatients. On request, one of the authors (SJS) provided expertise in the management of infectious diseases (ID). The current retrospective case series includes patients for whom ID consultation was requested over a 3-year period (from January 1, 2007, to December 8, 2009) and who ultimately received daptomycin for treatment of symptomatic VRE UTI. In summarizing the cases we managed for this report, we sought approval from the ethics committee of our institution ("the Saint Mary's Hospital IRB") (protocol 12-18-09). After IRB permission was granted, written informed consent was obtained from all patients for inclusion in this study and for publication of their medical information. A copy of the written consent is available for review by the editor of this journal.

A large majority of VRE-infected patients in our hospital are seen by an ID specialist because of intrinsic limitations in the treatment options and the hospital requirement of gown-and-glove contact isolation procedures for these patients. For this retrospective case series, patients were identified for consultation after a telephone call about a VRE-positive urine culture. To be treated with daptomycin and included in this analysis, patients had to exhibit urinary symptoms, pyuria $(>5$ white blood cells per high-power field), or both on urinalysis with a positive VRE culture.

VRE susceptibility was determined according to a VITEK 2 (bioMérieux, Inc, Durham, NC) microbial identification system, with daptomycin susceptibility determined by Etest (bioMérieux, Inc) on request. Patients who had positive VRE urinary cultures without significant pyuria on urinalysis were considered colonized rather than infected and, therefore, were not included in this analysis.

Once a patient was determined to have a VRE UTI, screening creatinine values were measured in all cases, and creatine phosphokinase (CPK) levels were determined in most cases. Daptomycin was generally administered at a planned dosage regimen of $\geq 5 \mathrm{mg} / \mathrm{kg}$ every 24 hours (if creatinine clearance $[\mathrm{CrCl}] \geq 30 \mathrm{~mL} / \mathrm{min}$ ) or every 48 hours (if $\mathrm{CrCl}<30 \mathrm{~mL} / \mathrm{min}$ ). Dosage was determined based on actual body weight for all patients in this analysis. A dose of $\geq 5 \mathrm{mg} / \mathrm{kg}$ was chosen to provide ample urinary concentrations of the drug.

Clinical cure was defined by the resolution of symptoms in the best judgment of the ID clinician involved in the care of the patient (SJS). These included urinary symptoms such as dysuria, urinary frequency, and changes in urine character. Other symptoms such as abdominal pain, fever, malaise, and anorexia were also monitored for improvement. Follow-up urinalysis and urine culture were typically performed at the end of the daptomycin treatment course. The presence or absence of pyuria in urinalysis at the end of treatment was not in itself a strict criterion for clinical cure because it might have resulted from bladder irritation, drugs, or other causes. Regardless of whether pyuria was initially present 
and remained present at the end of the treatment course, microbiologic data were examined to help determine whether microbiologic cure was achieved. Microbiologic cure was defined by the eradication of VRE in urine cultures taken after completion of the daptomycin treatment course.

\section{Results}

Between January 1, 2007, and December 8, 2009, we identified 10 patients with VRE UTIs who were treated by daptomycin-based regimens at our institution. Baseline characteristics of these patients are shown in Table 1; all patients had cystitis. As is apparent from the medical histories, most of the VRE UTIs were acquired in the hospital. In addition, the patients had a variety of risk factors typical for VRE infections, such as prolonged hospital stays, multiple previous UTIs, uropathy including the requirement for a long-term Foley catheter, and multiple past courses of antibiotics [7].

Table 2 presents the details of the daptomycin-based regimens for the management of VRE UTIs in our selected patient cases. Dosages used were based on our empirical experience because there are no recommendations in the literature with regard to daptomycin use in the treatment of patients with VRE UTIs. Because of the high therapeutic index and the fixed vial size for daptomycin (500 mg), some variability in actual perweight dosing occurred. The unusually high dose used in patient $3(13 \mathrm{mg} / \mathrm{kg}$ every 24 hours in a 30 -year-old man with quadriplegia) is explained by the concomitant management of a Staphylococcus spp. bacteremia.

Daptomycin-based courses of antibiotic treatment achieved clinical cure and successful eradication of VRE in all patients in this representative sample of a diverse patient population in our medical facility (Table 2). Indwelling catheters are common sites of infection, and similar results of daptomycin treatment were observed in patients with and without Foley catheters. Daptomycin was effective at eradicating VRE in patients regardless of whether they had had previous UTIs. Additionally, daptomycin eradicated VRE regardless of the level of renal function. Overall, daptomycin was well tolerated, and no reports of adverse events such as alteration of kidney function, muscle weakness or pain, and elevated levels of CPK enzymes were included. For most patients, CPK levels were measured at the time of daptomycin therapy initiation and once or twice more in the next 7 to 10 days. Even for the patient receiving the highest dose $(13 \mathrm{mg} / \mathrm{kg}$, patient 3$)$, no elevation was above the normal CPK range.

\section{Discussion}

The data in this case series show daptomycin to be a safe and effective therapeutic option in the treatment of patients with VRE UTIs. Limitations of the present study include the fact that this is a retrospective case series with a small number of patients and clinical records that are often limited and variable. Furthermore, because of the paucity of data in the literature, it is unclear what the most appropriate daptomycin dose is for the treatment of VRE UTIs. Nevertheless, it is clear that VRE are increasingly involved in nosocomial infections. Of concern, VRE may transfer vancomycin resistance to other bacterial species, including S. aureus [13]. Uncontrolled dissemination of VRE infections within health care institutions has been facilitated by incautious contact with contaminated medical equipment and surfaces, colonized health care personnel, and infected patients [14]. For example, the spread of a strain of linezolid-resistant VRE has been reported in one zone of a transplantation unit despite extensive precautions [15].

Another major factor that has led to the spread of VRE infection is poor infection control techniques that rely on cephalosporins and other antibacterials inactive against enterococci [8]. A recent report [7] found that more than $30 \%$ of all clinical isolates of Enterococcus were resistant to vancomycin, including more than $90 \%$ of E. faecium isolates. For these reasons, appropriate antibiotic susceptibility testing is a mainstay of good clinical practice to limit the spread of multidrugresistant strains, and agents that have specific activity against these strains must be used to eradicate these difficult-to-treat infections.

The urinary tract is one of the main portals for entry of VRE, so it is hardly surprising that the urinary tract is a major site of infection [13]. Management of VRE UTIs usually requires correction of any factors contributing to the infection. Catheter removal, obstruction relief, abscess drainage, and initiation of antimicrobial therapy are all initial steps taken in clinical practice [7].

In this case series, patients with VRE UTIs were treated with daptomycin, a cyclic lipopeptide antibiotic that has rapid bactericidal activity against a variety of Gram-positive pathogens [16]. Daptomycin acts by binding to bacterial cell membranes and inducing rapid depolarization, which inhibits DNA, RNA, and protein synthesis and leads to cell death [10]. In the 10 cases described here, daptomycin was chosen as the treatment option because of its efficacy profile, sensitivity testing, and low resistance rates for both major species of Enterococcus.

Another important consideration when appropriate treatment for VRE UTI is chosen is the presence of intact drug at the site of infection [16]. Daptomycin is eliminated primarily by the kidney; approximately $52 \%$ is excreted unchanged into the urine after intravenous administration [10]. Other agents active against VRE, such as linezolid, quinupristin-dalfopristin, and tigecycline, have a lower fraction of urinary excretion [7], which may 
Table 1 Selected baseline clinical characteristics of patients infected with VRE UTIs selected for daptomycin therapy

\begin{tabular}{|c|c|c|c|c|c|c|c|c|c|c|}
\hline Patient & $\begin{array}{l}\text { Age } \\
\text { (y)/Sex }\end{array}$ & $\begin{array}{l}\text { Weight } \\
(\mathrm{kg})\end{array}$ & Medical history & $\begin{array}{l}\text { Previous } \\
\text { UTIs }\end{array}$ & $\begin{array}{l}\text { Foley } \\
\text { catheter }\end{array}$ & $\begin{array}{l}\mathrm{Cr}(\mathrm{mg} / \mathrm{dL}) / \\
\mathrm{CrCl}(\mathrm{mL} / \mathrm{min})\end{array}$ & $\begin{array}{l}\text { Urinalysis } \\
\text { (WBC/hpf) }\end{array}$ & VRE isolate & $\begin{array}{l}\text { VRE culture } \\
\text { (CFU/mL) }\end{array}$ & Susceptibilities \\
\hline \multicolumn{11}{|c|}{ Normal kidney function } \\
\hline 1 & $72 / F$ & 67 & $\begin{array}{l}\text { Long hospital stay after surgery for large mucinous } \\
\text { adenocarcinoma of the ovary; ADM: lethargy } \\
\text { thought secondary to sedative overdose }\end{array}$ & No & No & $0.6 / 90$ & $40-50$ & E. faecium & 50,000 & $\begin{array}{l}\text { R: AMP, LNZ, TET, VAN; } \\
\text { S: GEN, QD, TIG }\end{array}$ \\
\hline 2 & $61 / F$ & 107 & $\begin{array}{l}\text { MS, neurogenic bladder; ADM: MS flare, } \\
\text { Pseudomonas spp. UTI treated with CIP }\end{array}$ & Yes & Yes & $0.8 / 100$ & $60-65$ & E. faecium & 30,000 & R: PCN, TET, VAN; I: NFT; S: DAP \\
\hline 3 & 30/M & 66 & $\begin{array}{l}\text { C5 quadriplegia, neurogenic bladder, recent UTIs } \\
\text { caused by Pseudomonas aeruginosa; ADM: abdominal } \\
\text { pain, cloudy urine, treated empirically with TOB }\end{array}$ & Yes & No & $0.2 />120$ & $>100$ & E. faecalis & 100,000 & R: TET, VAN; S: DAP, PCN \\
\hline \multicolumn{11}{|c|}{ Mild kidney disease (CKD 2) } \\
\hline 4 & $70 / F$ & 66 & $\begin{array}{l}\text { Neurogenic bladder, nephrolithiasis; ADM: lethargy, } \\
\text { hypotension }\end{array}$ & Yes & Yes & $0.6 / 83$ & $25-30$ & E. faecium & $>100,000$ & R: AMP, PCN, VAN \\
\hline 5 & $67 / F$ & 109 & $\begin{array}{l}\text { ADM: acute on chronic respiratory failure due to } \mathrm{CHF} \text {, } \\
\text { hypoventilation secondary to obesity; initially required } \\
\text { intubation and ventilation in the ICU, developed fever } \\
\left(101^{\circ} \mathrm{F}\right) \text {; initially unspecified Enterococcus infection } \\
\text { treated with VAN and CEF }\end{array}$ & No & No & $1.4 / 63$ & NP & E. faecium & 5000 & R: AMP, NFT, VAN \\
\hline \multicolumn{11}{|c|}{ Moderate kidney disease (CKD 3) } \\
\hline 6 & $83 / F$ & 55 & Acute exacerbation of COPD & No & No & $1.1 / 34$ & TNTC & E. faecium & $>100,000$ & R: PCN, TET, VAN; I: NFT \\
\hline 7 & $83 / F$ & 77 & $\begin{array}{l}\text { Stays at a nursing home; recently hospitalized for } \\
\text { stenting of right femoral artery for vascular disease }\end{array}$ & No & No & $1.2 / 46$ & $25-50$ & E. faecium & 40,000 & R: NFT, PCN, TET, VAN; S: DAP \\
\hline 8 & 86/M & 70 & $\begin{array}{l}\text { CKD stage } 3 \text {, nephrolithiasis, obstructive uropathy with } \\
\text { benign prostatic hypertrophy, recent UTI caused by } \\
\text { E. coli and } P \text {. aeruginosa; ADM: bloody urine, initial } \\
\text { evaluation revealed infection with } E \text {. coli and } P \text {. aeruginosa, } \\
\text { treated with CEF }\end{array}$ & Yes & Yes & $1.1 / 43$ & $10-15$ & E. faecium & 80,000 & R: PCN, TET, VAN \\
\hline \multicolumn{11}{|c|}{ Severe kidney disease/kidney failure (CKD 4-5) } \\
\hline 9 & $90 / F$ & 60 & $\begin{array}{l}\text { Severe dementia, stays at extended-care facility; ADM: } \\
\text { decreased oral intake, abdominal pain, fever }\left(100.9^{\circ} \mathrm{F}\right)\end{array}$ & Yes & No & $1.1 / 26$ & TNTC & E. faecium & $>100,000$ & R: AMP, CIP, NFT, TET, VAN \\
\hline 10 & $60 / M$ & 81 & $\begin{array}{l}\text { Liver transplant for hepatitis C cirrhosis; ADM: chest pain, } \\
\text { E. coli empyema complicating previous CABG surgery }\end{array}$ & No & No & $4.1 / 19$ & NP & E. faecium & 100,000 & R: DOX, PCN, VAN \\
\hline
\end{tabular}

ADM admission, AMP ampicillin, CABG coronary artery bypass graft, CEF ceftazidime, CFU colony-forming units, CHF congestive heart failure, CIP ciprofloxacin, CKD chronic kidney disease, COPD chronic obstructive pulmonary disease, $\mathrm{Cr}$ creatinine, $\mathrm{CrCl}$ creatinine clearance, DAP daptomycin, DOX doxycycline, GEN gentamicin, I intermediate, ICU intensive care unit, LNZ linezolid, MS multiple sclerosis, NFT nitrofurantoin, NP no performed, $P C N$ penicillin, $Q D$ quinupristin-dalfopristin, $R$ resistant, $S$ susceptible, TET tetracycline, TIG tigecycline, TNTC too numerous to count, TOB tobramycin, UTI urinary tract infection, VAN vancomycin, WBC/hpf white blood cells per high-power field. 
Table 2 Details of management of VRE UTIs and outcome of daptomycin course

\begin{tabular}{|c|c|c|c|c|}
\hline Patient & Daptomycin dosing & Daptomycin treatment duration & Concomitant antibiotics & Posttreatment urine culture \\
\hline \multicolumn{5}{|c|}{ Normal kidney function } \\
\hline 1 & $7.5 \mathrm{mg} / \mathrm{kg}$ q24h & 7 days & None & VRE eradicated \\
\hline 2 & $8 \mathrm{mg} / \mathrm{kg} \mathrm{q} 24 \mathrm{~h}$ & 3 days & None & VRE eradicated \\
\hline 3 & $13 \mathrm{mg} / \mathrm{kg} \mathrm{q} 24 \mathrm{~h}^{*}$ & 10 days & None & VRE eradicated \\
\hline \multicolumn{5}{|c|}{ Mild kidney disease (CKD 2) } \\
\hline 4 & $5 \mathrm{mg} / \mathrm{kg} \mathrm{q} 24 \mathrm{~h}$ & 3 days & Fluconazole & VRE eradicated \\
\hline 5 & $5 \mathrm{mg} / \mathrm{kg} \mathrm{q} 24 \mathrm{~h}$ & 3 days & None & VRE eradicated \\
\hline \multicolumn{5}{|c|}{ Moderate kidney disease (CKD 3) } \\
\hline 6 & $5 \mathrm{mg} / \mathrm{kg} \mathrm{q} 24 \mathrm{~h}$ & 3 days & None & VRE eradicated \\
\hline 7 & $5 \mathrm{mg} / \mathrm{kg}$ q24h & 3 days & Gentamicin 120 mg once a day & $\begin{array}{l}5 \text { days after initiation of daptomycin; } \\
\text { VRE eradicated }\end{array}$ \\
\hline 8 & $5 \mathrm{mg} / \mathrm{kg} \mathrm{q} 24 \mathrm{~h}$ & 3 days & None & VRE eradicated \\
\hline \multicolumn{5}{|c|}{ Severe kidney disease/kidney failure (CKD 4-5) } \\
\hline 9 & $5 \mathrm{mg} / \mathrm{kg} \mathrm{q} 24 \mathrm{~h}^{\dagger}$ & 3 days & None & VRE eradicated \\
\hline 10 & $5 \mathrm{mg} / \mathrm{kg} \mathrm{q} 48 \mathrm{~h}$ & 3 days & None & $\begin{array}{l}\text { VRE eradicated; patient died } 2 \text { days } \\
\text { later (not related to infection) }\end{array}$ \\
\hline
\end{tabular}

CKD chronic kidney disease, $q 24 h$ once every 24 hours, $q 48 h$ once every 48 hours, VRE vancomycin-resistant enterococci.

As determined using the Cockcroft-Gault equation, CKD $2=$ glomerular filtration rate (GFR) of $60-89 \mathrm{~mL} / \mathrm{min} / 1.73 \mathrm{~m}{ }^{2}, \mathrm{CKD} 3=\mathrm{GFR}$ of $30-59 \mathrm{~mL} / \mathrm{min} / 1.73 \mathrm{~m}{ }^{2}$, and CKD 4-5 = GFR of $<30 \mathrm{~mL} / \mathrm{min} / 1.73 \mathrm{~m}^{2}$.

Follow-up durations: microbiologic assessment (urine culture) 3 days, clinical assessments up to $\sim 7$ days posttreatment.

* Use of unusually high per-weight dose was recommended by the ID specialist for the concomitant management of Staphylococcus spp. bacteremia.

${ }^{+} \mathrm{ID}$ consult resulted in clinical decision to treat $\mathrm{q} 24 \mathrm{~h}$ rather than $\mathrm{q} 48 \mathrm{~h}$ for more rapid results in this patient.

potentially limit their effectiveness in the management of VRE UTIs.

Most strains of VRE are resistant to penicillin and ampicillin, although higher-dose ampicillin, doxycycline, and nitrofurantoin remain viable treatment options. More often, drug choices used to treat VRE include daptomycin, linezolid, quinupristin-dalfopristin, and tigecycline. These compounds have similarly low MIC values against VRE species [7]. All these antibiotics except daptomycin exhibit bacteriostatic properties; daptomycin is bactericidal [7]. Daptomycin has similar MIC values for E. faecium and $E$. faecalis [7] and is effective in treating either pathogen. Daptomycin resistance among VRE strains remains rare [11]; numerous global surveillance studies have demonstrated higher susceptibility levels in VRE strains using daptomycin than in strains using linezolid or quinupristindalfopristin [16]. Resistance to linezolid among VRE isolates has been well described and is of increasing clinical concern [16-19]. Quinupristin-dalfopristin has limited activity against $E$. faecalis [7]. In addition, vancomycinresistant E. faecium strains have shown emerging resistance to quinupristin-dalfopristin; $3.4 \%$ of urinary tract isolates were resistant in a study at 28 medical centers in the United States [3]. Use of quinupristin-dalfopristin is also limited by its potential systemic and infusion site-related adverse effects, including myalgia and arthralgia $[7,13]$.

Our experience confirms and extends the findings of a previous case series on the use of daptomycin for the treatment of VRE UTIs. The previous study [12] of 5 hospitalized patients with indwelling catheters and VRE UTIs who received 5-day courses of daptomycin showed that all patients achieved complete eradication of infection at daptomycin doses of 1.4 to $3.7 \mathrm{mg} / \mathrm{kg}$ daily. Taken together, these case studies emphasize the importance of further analyses to delineate the appropriate doses of daptomycin for the treatment of patients with VRE UTIs.

\section{Conclusions}

In the current case series, daptomycin was shown to be a safe and effective therapeutic option in the management of VRE UTIs. Because of the increasing prevalence of VRE infection and the limited treatment options available, we anticipate that management of VRE UTIs will continue to be challenging for the clinician. Based on our experience, we believe that daptomycin is a valuable treatment option for problematic UTIs. These findings must be confirmed in larger randomized clinical trials. However, despite the lack of data about therapeutic options for VRE UTIs and the intrinsic limitations of descriptive case reports, our positive experience with daptomycin may be of value to the clinical community.

\section{Abbreviations}

CPK: Creatinine phosphokinase; $\mathrm{CrCl}$ : Creatinine clearance; FDA: Food and

Drug Administration; ID: Infectious disease; IRB: Institutional review board;

MIC: Minimal inhibitory concentration; UTI: Urinary tract infection;

VRE: Vancomycin-resistant enterococci. 


\section{Competing interests}

DPR and SJS have no competing interests to report. MA-G is an employee of Cubist Pharmaceuticals, Inc. The authors received no financial support or honoraria for the development of this manuscript.

\section{Authors' contributions}

DPR and SJS conceived of the study, collected and analyzed data from patient records, wrote the first draft of the manuscript, and reviewed all subsequent drafts. MA-G contributed to data analysis and interpretation and reviewed all drafts. All authors read and approved the final manuscript.

\section{Acknowledgements}

Julien Ferrari, PhD, and Thomas Rotolo, PhD, of ApotheCom provided assistance in drafting and editing the manuscript. Cubist Pharmaceuticals, Inc., supported the services provided by ApotheCom.

\section{Author details}

${ }^{1}$ Department of Internal Medicine, St. Mary's Hospital, Waterbury, Connecticut 06706, USA. ${ }^{2}$ Cubist Pharmaceuticals, Inc., Lexington, Massachusetts 02421 USA. ${ }^{3}$ Division of Infectious Diseases, St. Mary's Hospital, Waterbury, Connecticut 06706, USA. ${ }^{4}$ Current affiliations: Toledo Hospital, Toledo, Ohio 43606, USA. ${ }^{5}$ Current affiliations: Cornell Scott Hill Health Center, New Haven, Connecticut 06519, USA

Received: 14 November 2012 Accepted: 9 July 2013

Published: 16 July 2013

\section{References}

1. Reik R, Tenover FC, Klein E, McDonald LC: The burden of vancomycinresistant enterococcal infections in US hospitals, 2003 to 2004. Diagn Microbiol Infect Dis 2008, 62:81-85.

2. Hidron Al, Edwards JR, Patel J, Horan TC, Sievert DM, Pollock DA, Fridkin SK: NHSN annual update: antimicrobial-resistant pathogens associated with healthcare-associated infections: annual summary of data reported to the National Healthcare Safety Network at the Centers for Disease Control and Prevention, 2006-2007. Infect Control Hosp Epidemiol 2008, 29:996-1011.

3. Zhanel GG, Laing NM, Nichol KA, Palatnick LP, Noreddin A, Hisanaga T, Johnson JL, Hoban DJ: Antibiotic activity against urinary tract infection (UTI) isolates of vancomycin-resistant enterococci (VRE): results from the 2002 North American Vancomycin Resistant Enterococci Susceptibility Study (NAVRESS). J Antimicrob Agents Chemother 2003, 52:382-388.

4. Deshpande LM, Fritsche TR, Moet GJ, Biedenbach DJ, Jones RN: Antimicrobial resistance and molecular epidemiology of vancomycinresistant enterococci from North America and Europe: a report from the SENTRY antimicrobial surveillance program. Diagn Microbiol Infect Dis 2007, 58:163-170.

5. Ramsey AM, Zilberberg MD: Secular trends of hospitalization with vancomycin-resistant enterococcus infection in the United States, 2000-2006. Infect Control Hosp Epidemiol 2009, 30:184-186.

6. Wisplinghoff $H$, Bischoff $T$, Tallent SM, Seifert $H$, Wenzel RP, Edmond MB: Nosocomial bloodstream infections in US hospitals: analysis of 24,179 cases from a prospective nationwide surveillance study. Clin Infect Dis 2004, 39:309-317.

7. Heintz BH, Halilovic J, Christensen CL: Vancomycin-resistant enterococcal urinary tract infections. Pharmacotherapy 2010, 30:1136-1149.

8. Rice LB: Emergence of vancomycin-resistant enterococci. Emerg Infect Dis 2001, 7:183-187.

9. Rybak MJ, Hershberger E, Moldovan T, Grucz RG: In vitro activities of daptomycin, vancomycin, linezolid, and quinupristin-dalfopristin against Staphylococci and Enterococci, including vancomycin-intermediate and -resistant strains. Antimicrob Agents Chemother 2000, 44:1062-1066

10. Cubicin ${ }^{\oplus}$ (daptomycin for injection). Lexington, MA: Cubist Pharmaceuticals, Inc.; 2013

11. Sader HS, Jones RN: Antimicrobial susceptibility of gram-positive bacteria isolated from US medical centers: results of the daptomycin surveillance program (2007-2008). Diagn Microbiol Infect Dis 2009, 65:158-162.

12. Fisher L, North D: Effectiveness of low-dose daptomycin in the treatment of vancomycin-resistant enterococcal urinary tract infections. Int J Antimicrob Agents 2009, 33:493-494.

13. Zirakzadeh A, Patel R: Vancomycin-resistant enterococci: colonization, infection, detection, and treatment. Mayo Clin Proc 2006, 81:529-536.
14. Karanfil LV, Murphy M, Josephson A, Gaynes R, Mandel L, Hill BC, Swenson JM: A cluster of vancomycin-resistant Enterococcus faecium in an intensive care unit. Infect Control Hosp Epidemiol 1992, 13:195-200.

15. Herrero IA, Issa NC, Patel R: Nosocomial spread of linezolid-resistant, vancomycin-resistant Enterococcus faecium. N Engl J Med 2002, 346:867-869.

16. Canton R, Ruiz-Garbajosa P, Chaves RL, Johnson AP: A potential role for daptomycin in enterococcal infections: what is the evidence? J Antimicrob Agents Chemother 2010, 65:1126-1136.

17. Scheetz MH, Knechtel SA, Malczynski M, Postelnick MJ, Qi C: Increasing incidence of linezolid-intermediate or -resistant, vancomycin-resistant Enterococcus faecium strains parallels increasing linezolid consumption. Antimicrob Agents Chemother 2008, 52:2256-2259.

18. Schulte B, Heininger A, Autenrieth IB, Wolz C: Emergence of increasing linezolid-resistance in enterococci in a post-outbreak situation with vancomycin-resistant Enterococcus faecium. Epidemiol Infect 2008, 136:1131-1133.

19. Pogue JM, Paterson DL, Pasculle AW, Potoski BA: Determination of risk factors associated with isolation of linezolid-resistant strains of vancomycin-resistant Enterococcus. Infect Control Hosp Epidemiol 2007, 28:1382-1388.

doi:10.1186/1471-2490-13-33

Cite this article as: Ramaswamy et al:: Use of daptomycin in the treatment of vancomycin-resistant enterococcal urinary tract infections: a short case series. BMC Urology 2013 13:33.

\section{Submit your next manuscript to BioMed Central and take full advantage of:}

- Convenient online submission

- Thorough peer review

- No space constraints or color figure charges

- Immediate publication on acceptance

- Inclusion in PubMed, CAS, Scopus and Google Scholar

- Research which is freely available for redistribution 